\title{
Positive Solutions for Fractional Differential Equations with Multi-Point Boundary Value Problems
}

\author{
Lina Zhou' ${ }^{1}$, Weihua Jiang ${ }^{2}$ \\ ${ }^{1}$ College of Mathematics and Information Science, Hebei Normal University, Shijiazhuang, China \\ ${ }^{2}$ College of Science, Hebei University of Science and Technology, Shijiazhuang, China \\ Email: Inazhou@163.com, xytzIn@gmail.com
}

Received November 2013

\begin{abstract}
In this paper, a fractional multi-point boundary value problem is considered. By using the fixed point index theory and Krein-Rutman theorem, some results on existence are obtained.
\end{abstract}

\section{Keywords}

\section{Caputo Fractional Derivative, Fractional Integral, Boundary Value Problem, Fixed Point Index} Theory

\section{Introduction}

Fractional differential equations have been of great interest recently. This is due to the intensive development of the theory of fractional calculus itself as well as its applications. Apart from diverse areas of mathematics, fractional differential equations arise in rheology, dynamical processes in self similar and porous structures, electrical networks, visco-elasticity, chemical physics, and many other branches of science. For details, see [1]-[7].

It should be noted that most of papers and books on fractional calculus are devoted to the solvability of linear initial fractional differential equations on terms of special functions. Recently, there are some papers dealing with the existence and multiplicity of solution to the nonlinear fractional differential equations boundary value problems, see [8]-[14].

Zhao [11] investigated the existence and uniqueness of positive solutions for a local boundary value problem of fractional differential equation.

$$
\left\{\begin{array}{c}
D_{0+}^{\alpha} u(t)+f(t, u(t))=0 \quad 0<t<1 \\
u^{\prime}(0)-\beta u(\xi)=0, u^{\prime}(1)+\gamma u(\eta)=0
\end{array}\right.
$$

where $\alpha$ is a real number with $1<\alpha \leq 2,0 \leq \beta, \gamma \leq 1,0 \leq \xi \leq \eta \leq 1, D_{0+}^{\alpha}$ is the Caputo's derivative.

Inspired by above work, we will consider the fractional boundary value problem 


$$
\left\{\begin{array}{c}
D_{0+}^{\alpha} u(t)+f(t, u(t))=0 \quad 0<t<1 \\
u^{\prime}(0)-\beta u(\xi)=0, u^{\prime}(1)+\sum_{i=1}^{m-3} \gamma_{i} u\left(\eta_{i}\right)=0
\end{array}\right.
$$

where $\alpha$ is a real number with $1<\alpha \leq 2,0 \leq \beta \leq 1,0 \leq \gamma_{i} \leq 1, i=1,2, \cdots, m-3, \quad 0 \leq \xi<\eta_{1}<\eta_{2}<\cdots<\eta_{m-3} \leq 1$, $D_{0+}^{\alpha}$ is the Caputo's derivative.

Let $\Delta=\beta\left(1+\sum_{i=1}^{m-3} \gamma_{i} \eta_{i}\right)+\sum_{i=1}^{m-3} \gamma_{i}(1-\beta \xi)$.

Now we list some conditions for convenience.

(H1) $(\alpha-1)(1-\beta \xi)>\Delta, 1+\sum_{i=1}^{m-3} \gamma_{i}\left(\eta_{i}-1\right)>0$

(H2) $f:[0,1] \times R^{+} \rightarrow R^{+}$satisfied Carathéodory condition,that is $f(\cdot, u)$ is measurable for each fixed $u \in R^{+}$and $f(t, \cdot)$ is continuous for a.e. $t \in[0,1]$. For any $r>0$, there existed $\Phi(t) \in L^{1}[0,1]$ such that $f(t, u) \leq \Phi(t)$, where $u \in[0, r]$; a.e. $t \in[0,1]$.

(H3) $\forall l>0, \exists L>0,|f(t, u)|<L$ where $u \in[0, l]$; a.e. $t \in[0,1]$.

\section{Preliminary}

For the convenience of readers, we provide some background material in this section. \{ \bf Definition 2.1[7] The Riemann-Liouville fractional of order $\alpha$ for function $y$ is defined as

$$
I_{0+}^{\alpha} y(t)=\frac{1}{\Gamma(\alpha)} \int_{0}^{t}(t-s)^{\alpha-1} y(s) d s
$$

Definition 2.2 [7] The Caputo's derivative for function $y$ is defined as

$$
D_{0+}^{\alpha} y(t)=\frac{1}{\Gamma(n-\alpha)} \int_{0}^{t} \frac{y^{(n)}(s)}{(t-s)^{\alpha+1-n}} d s
$$

Lemma 2.1 [14] Let $\alpha>0$, then the fractional differential equation $D_{0+}^{\alpha} u(t)=0$

has solutions $u(t)=c_{1}+c_{2} t+c_{3} t^{2}+\cdots+c_{n} t^{n-1}, c_{i} \in R, i=1,2, \cdots, n, n=[\alpha]+1$

Lemma 2.2 [14] Let $\alpha>0$, then for some $c_{i} \in R, i=1,2, \cdots, n, n=[\alpha]+1$

Lemma 2.3 (Krein-Rutman) [15] Let $K$ be a reproducing cone in a real Banach space $X$ and let $L: X \rightarrow X$ be a compact linear operator with $L(K) \subseteq K . r(L)$ is the spectral radius of $L$ If $r(L)>0$, then there exists $\varphi_{1} \in K \backslash\{0\}$ such that $L \varphi_{1}=r(L) \varphi_{1}$.

Lemma 2.4 [16] Let $X$ is a Banach space, $P$ be a cone in $X$ and $\Omega(P)$ be a bounded open subset in $P$.

Suppose that $A: \overline{\Omega(P)} \rightarrow P$ is a completely continuous operator. Then the following results hold:

(1) If there exists $u_{0} \in P \backslash\{0\}$ such that $u \neq A u+\lambda u_{0}, \forall u \in \partial \Omega(P), \lambda>0$ then the fixed point index $i(A, \Omega(P), P)=0$.

(2) If $0 \in \Omega(P)$ and $A u \neq \lambda u, \forall u \in \partial \Omega(P), \lambda \geq 1$, then the fixed point index $i(A, \Omega(P), P)=1$.

Take $X=C[0,1]$ with norm $\|x(t)\|=\max _{t \in[0,1]}|x(t)|, \quad Y=L^{1}[0,1]$ with norm $\|x(t)\|_{1}=\int_{0}^{1}|x(t)| d t$.

$K=\{u \in X \mid u(t) \geq 0, t \in[0,1]\}$ Obviously $K$ is a reproducing cone of $X$.

Lemma 2.5 If $y \in C[0,1], 1<\alpha \leq 2$, then the unique solution of

$$
\left\{\begin{array}{lr}
D_{0+}^{\alpha} u(t)+y(t)=0 & 0<t<1 \\
u^{\prime}(0)-\beta u(\xi)=0, u^{\prime}(1)+\sum_{i=1}^{m-3} \gamma_{i} u\left(\eta_{i}\right)=0
\end{array}\right.
$$

is $u(t)=\int_{0}^{1} G(t, s) y(s) d s$, where 


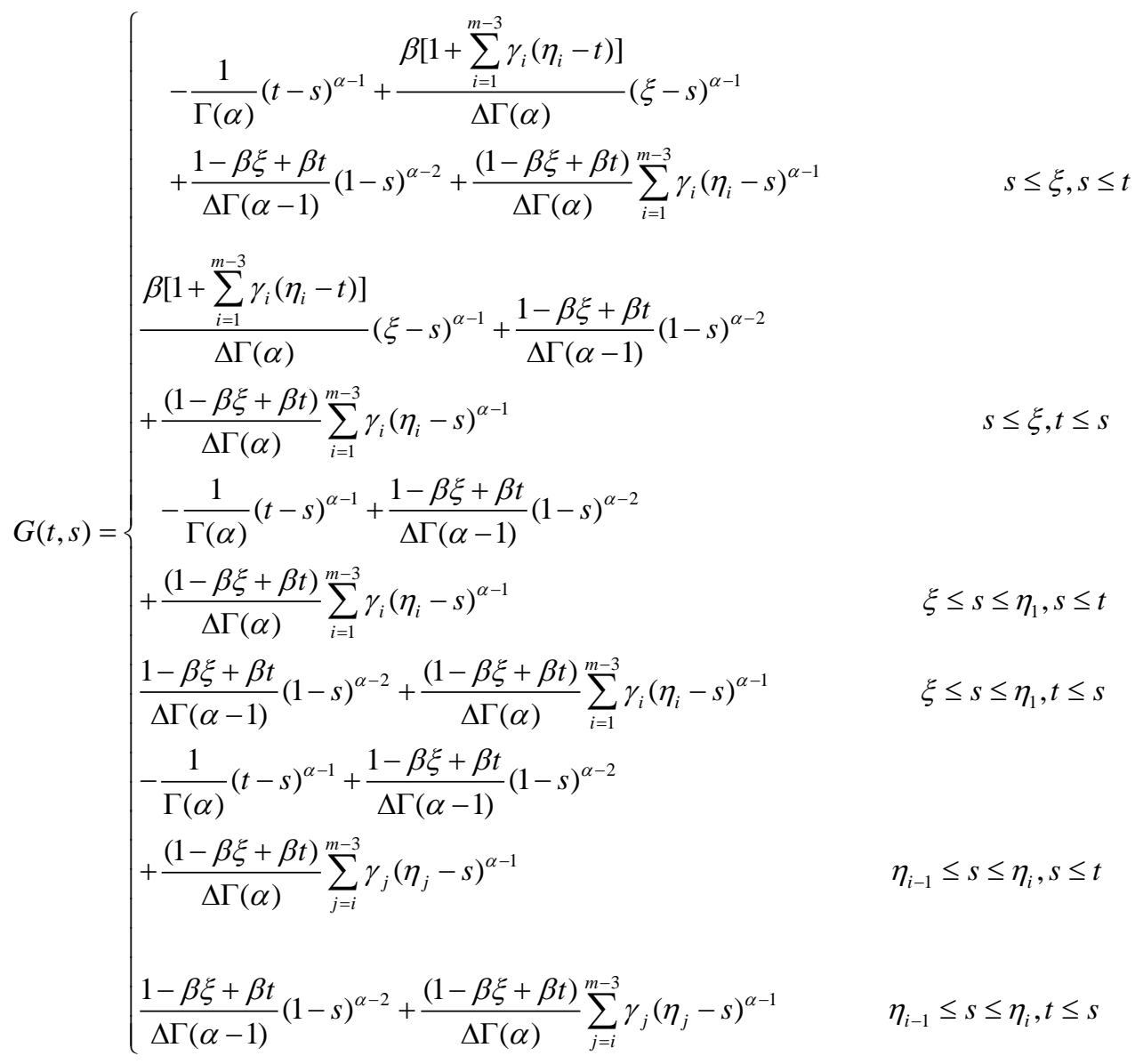

Proof: The equation $D_{0+}^{\alpha} u(t)+y(t)=0$ has a unique solution

$$
u(t)=-\frac{1}{\Gamma(\alpha)} \int_{0}^{t}(t-s)^{\alpha-1} y(s) d s+c_{1}+c_{2} t
$$

where $c_{1}, c_{2} \in R$. By $u^{\prime}(0)-\beta u(\xi)=0, u^{\prime}(1)+\sum_{i=1}^{m-3} \gamma_{i} u\left(\eta_{i}\right)=0$, we have

$$
\begin{gathered}
c_{1}=\frac{1}{\Delta}\left[\beta\left(1+\sum_{i=1}^{m-3} \gamma_{i} \eta_{i}\right) I_{0+}^{\alpha} y(\xi)+(1-\beta \xi) I_{0+}^{\alpha-1} y(1)+(1-\beta \xi) \sum_{i=1}^{m-3} \gamma_{i} I_{0+}^{\alpha} y\left(\eta_{i}\right)\right] \\
c_{2}=\frac{1}{\Delta}\left[\beta I_{0+}^{\alpha-1} y(1)+\beta \sum_{i=1}^{m-3} \gamma_{i} I_{0+}^{\alpha} y\left(\eta_{i}\right)-\beta \sum_{i=1}^{m-3} \gamma_{i} I_{0+}^{\alpha} y(\xi)\right] \\
u(t)=-\frac{1}{\Gamma(\alpha)} \int_{0}^{t}(t-s)^{\alpha-1} y(s) d s+\frac{\beta\left[1+\sum_{i=1}^{m-3} \gamma_{i}\left(\eta_{i}-t\right)\right]}{\Delta \Gamma(\alpha)} \int_{0}^{t}(\xi-s)^{\alpha-1} y(s) d s+\frac{1-\beta \xi+\beta t}{\Delta \Gamma(\alpha-1)} \int_{0}^{1}(1-s)^{\alpha-2} y(s) d s \\
+\frac{(1-\beta \xi+\beta t)}{\Delta \Gamma(\alpha)} \sum_{i=1}^{m-3} \gamma_{i} \int_{0}^{\eta_{i}}\left(\eta_{i}-s\right)^{\alpha-1} y(s) d s
\end{gathered}
$$

The proof is complete.

Lemma 2.6 If (H1) hold, then there exist a constant $M$ such that $0 \leq G(t, s) \leq M(1-s)^{\alpha-2}, t, s \in[0,1]$ Proof: Obviously $G(t, s) \geq 0$, 


$$
\begin{aligned}
\max _{0 \leq t \leq 1} G(t, s) & \leq \frac{\beta\left[1+\sum_{i=1}^{m-3} \gamma_{i}\left(\eta_{i}-t\right)\right]}{\Delta \Gamma(\alpha)}(\xi-s)^{\alpha-1}+\frac{1-\beta \xi+\beta t}{\Delta \Gamma(\alpha-1)}(1-s)^{\alpha-2}+\frac{(1-\beta \xi+\beta t)}{\Delta \Gamma(\alpha)} \sum_{i=1}^{m-3} \gamma_{i}\left(\eta_{i}-s\right)^{\alpha-1} \\
& \leq \frac{\beta\left[1+\sum_{i=1}^{m-3} \gamma_{i} \eta_{i}\right]}{\Delta \Gamma(\alpha)}(1-s)^{\alpha-2}+\frac{1-\beta \xi+\beta}{\Delta \Gamma(\alpha-1)}(1-s)^{\alpha-2}+\frac{(1-\beta \xi+\beta)}{\Delta \Gamma(\alpha)} \sum_{i=1}^{m-3} \gamma_{i}(1-s)^{\alpha-2} \\
& =\frac{\beta\left(1+\sum_{i=1}^{m-3} \gamma_{i} \eta_{i}\right)+(\alpha-1)(1-\beta \xi+\beta)+\sum_{i=1}^{m-3} \gamma_{i}(1-\beta \xi+\beta)}{\Delta \Gamma(\alpha)}(1-s)^{\alpha-2}
\end{aligned}
$$

Let $M=\frac{\beta\left(1+\sum_{i=1}^{m-3} \gamma_{i} \eta_{i}\right)+(\alpha-1)(1-\beta \xi+\beta)+\sum_{i=1}^{m-3} \gamma_{i}(1-\beta \xi+\beta)}{\Delta \Gamma(\alpha)}$. The proof is completed.

Define an operator $A: K \rightarrow K$ and a linear operator $T: X \rightarrow X$ as follows:

$$
\begin{gathered}
A u(t)=\int_{0}^{1} G(t, s) f(s, u(s)) d s \\
T u(t)=\int_{0}^{1} G(t, s) u(s) d s
\end{gathered}
$$

Then the fixed point $u \in K \backslash\{0\}$ of $A$ is the positive solutions of (1)

\section{Main Results}

In order to obtain our main results, we firstly present and prove some lemmas.

Lemma 3.1 If (H1)-(H3) hold, then $A: K \rightarrow K$ and $T: X \rightarrow X$ are completely continuous.

Proof: According to the Lebesgue Dominated Convergence Theorem and Lemma 2.6, we have $A: K \rightarrow K$ is uniformly bounded and equicontinuous. It follows from Ascoli-Arzela theorem that $A: K \rightarrow K$ is completely continuous.

By the same method, we can get that $T: X \rightarrow X$ is completely continuous also.

Lemma3.2 If (H1)-(H2) hold, then $r(T)>0 \quad(r$ is the spectral radius of $T)$

Proof: Take $u(t) \equiv 1$

$$
\begin{aligned}
& T u(t)=\int_{0}^{1} G(t, s) u(s) d s \\
& =-\frac{1}{\Gamma(\alpha)} \int_{0}^{t}(t-s)^{\alpha-1} d s+\frac{\beta\left[1+\sum_{i=1}^{m-3} \gamma_{i}\left(\eta_{i}-t\right)\right]}{\Delta \Gamma(\alpha)} \int_{0}^{t}(\xi-s)^{\alpha-1} d s+\frac{1-\beta \xi+\beta t}{\Delta \Gamma(\alpha-1)} \int_{0}^{1}(1-s)^{\alpha-2} d s \\
& \quad+\frac{(1-\beta \xi+\beta t)}{\Delta \Gamma(\alpha)} \sum_{i=1}^{m-3} \gamma_{i} \int_{0}^{\eta_{i}}\left(\eta_{i}-s\right)^{\alpha-1} d s \\
& =-\frac{1}{\Gamma(\alpha)} \frac{t^{\alpha}}{\alpha}+\frac{\beta\left[1+\sum_{i=1}^{m-3} \gamma_{i}\left(\eta_{i}-t\right)\right]}{\Delta \Gamma(\alpha)} \frac{\xi^{\alpha}}{\alpha}+\frac{1-\beta \xi+\beta t}{\Delta \Gamma(\alpha-1)} \frac{1}{\alpha-1}+\frac{(1-\beta \xi+\beta t)}{\Delta \Gamma(\alpha)} \frac{\sum_{i=1}^{m-3} \gamma_{i} \eta_{i}^{\alpha}}{\alpha} \\
& \geq-\frac{1}{\Gamma(\alpha+1)}+\frac{1-\beta \xi}{\Delta \Gamma(\alpha)}+\frac{(1-\beta \xi)}{\Delta \Gamma(\alpha+1)} \sum_{i=1}^{m-3} \gamma_{i} \eta_{i}^{\alpha}:=l \\
& T^{2} u(t)=T(T u(t)) \geq T(l) \geq l^{2}, T^{n}(u(t)) \geq l^{n} \\
& \quad\left\|T^{n}\right\|^{\frac{1}{n}} \geq l, r(T)=\lim _{n \rightarrow \infty}\left\|T^{n}\right\|^{\frac{1}{n}} \geq l>0
\end{aligned}
$$

The proof is completed.

By Lemma 2.3, we can get there exists $\varphi_{0} \in K \backslash\{0\}$ such that $T \varphi_{0}=r(T) \varphi_{0}$ 
Define $f_{0}=\liminf _{u \rightarrow 0} \inf _{t \in[0,1] \backslash E} \frac{f(t, u)}{u}, f_{\infty}=\limsup _{u \rightarrow \infty} \sup _{t \in[0,1] E} \frac{f(t, u)}{u}$, where $E \subset[0,1]$, with $m(E)=0 \quad(m(E)$ is the lebesgue measure of $E$ and the same as follows).

Set $K_{\rho}=\{u \in K \mid\|u\|<\rho\}, \mu=\frac{1}{r(T)}$.

Lemma 3.3 Suppose $\mu<f_{0} \leq \infty$, then there exists $\rho_{0}>0$ such that for $\rho \in\left(0, \rho_{0}\right]$,

if $u \neq A u, \forall u \in \partial K_{\rho}$,then $i\left(A, K_{\rho}, K\right)=0$.

Proof: It follows from $\mu<f_{0}$ that there exists $\varepsilon>0$ and $\rho_{0}>0$ such that for a.e. $t \in[0,1], 0 \leq u \leq \rho_{0}$

$$
f(t, u) \geq(\mu+\varepsilon) u
$$

For $0<\rho \leq \rho_{0}$, assume $u \neq A u, \forall u \in \partial K_{\rho} \quad$ By Lemma 2.4, we need only to prove that

$$
u \neq A u+\lambda \varphi_{0}, \forall u \in \partial K_{\rho}, \lambda>0
$$

where $\varphi_{0} \in K \backslash\{0\}, T \varphi_{0}=r(T) \varphi_{0}$.

Otherwise, there exists $u_{0} \in \partial K_{\rho}, \lambda_{0}>0$ such that

$$
u_{0}=A u_{0}+\lambda_{0} \varphi_{0}
$$

Then $u_{0} \geq A u_{0}, u_{0} \geq \lambda_{0} \varphi_{0}$, by (2), we can get that

$$
A u_{0}=\int_{0}^{1} G(t, s) f\left(s, u_{0}(s)\right) d s \geq(\mu+\varepsilon) T u_{0}
$$

Considering $u_{0} \geq \lambda_{0} \varphi_{0}$, we get $A u_{0} \geq(\mu+\varepsilon) \lambda_{0} T \varphi_{0}>\lambda_{0} \varphi_{0}$.

This together with (3) means that $u_{0} \geq 2 \lambda_{0} \varphi_{0}$, by (4) we get $A u_{0} \geq 2 \lambda_{0} \varphi_{0}$. So $u_{0} \geq 3 \lambda_{0} \varphi_{0}$.

Repeating this process, we get that $u_{0} \geq n \lambda_{0} \varphi_{0}$, so we have $\left\|u_{0}\right\| \geq n \lambda_{0}\left\|\varphi_{0}\right\| \rightarrow \infty, n \rightarrow \infty$. This is a contradiction.

It follows from Lemma 2.4 that $i\left(A, K_{\rho}, K\right)=0, \rho \in\left(0, \rho_{0}\right]$. The proof is completed.

Lemma 3.4 Suppose $0 \leq f_{\infty}<\mu$, then there exists $r_{0}>0$ such that $i\left(A, K_{r}, K\right)=1$, for each $r>r_{0}$

Proof: Let $\varepsilon>0$ satisfy $f_{\infty}<\mu-\varepsilon$, then there exists $r_{1}>0$ such that

$$
f(t, u) \leq(\mu-\varepsilon) u \text {, for } u>r_{1} \text {, a.e. } t \in[0,1]
$$

By (H3),there existed $\Phi(t) \in L^{1}[0,1]$ such that $f(t, u) \leq \Phi(t)$, where $u \in\left[0, r_{1}\right]$; a.e. $t \in[0,1]$.

Thus, for all $u \in R^{+}$a.e. $t \in[0,1]$

$$
f(t, u) \leq(\mu-\varepsilon) u+\Phi(t)
$$

Since $\mu=\frac{1}{r(T)},\left(\frac{I}{\mu-\varepsilon}-T\right)^{-1}$ exists. Let $C=\left\|\int_{0}^{1} G(t, s) \Phi(s) d s\right\|, r_{0}=\left\|\left(\frac{I}{\mu-\varepsilon}-T\right)^{-1} \frac{C}{\mu-\varepsilon}\right\|$

Take $r>r_{0}$, we will show $A u \neq \lambda u$, for each $u \in \partial K_{r}, \lambda \geq 1$.

Otherwise, there exist $u_{0} \in \partial K_{r}, \lambda_{0} \geq 1$, such that $A u_{0}=\lambda_{0} u_{0}$, This together with (5), implies $u_{0} \leq \lambda_{0} u_{0}=A u_{0} \leq(\mu-\varepsilon) T u_{0}+C$ Then $\left(\frac{I}{\mu-\varepsilon}-T\right) u_{0}(t) \leq \frac{C}{\mu-\varepsilon}$. So we get $\frac{C}{\mu-\varepsilon}-\left(\frac{I}{\mu-\varepsilon}-T\right) u_{0}(t) \in K$. It follows from $\left(\frac{I}{\mu-\varepsilon}-T\right)^{-1}=\sum_{n=0}^{\infty}(\mu-\varepsilon)^{n+1} T^{n}$ and $T(K) \subset K$, we get $u_{0}(t) \leq\left(\frac{I}{\mu-\varepsilon}-T\right)^{-1} \frac{C}{\mu-\varepsilon}$. Therefore, we have $\left\|u_{0}\right\| \leq r_{0} \leq r$. This is a contradiction.

By Lemma 2.4 (2), we get $i\left(A, K_{r}, K\right)=1$, for each $r>r_{0}$. The proof is completed.

Theorem 3.1 Suppose $\mu<f_{0} \leq \infty$ and $0 \leq f_{\infty}<\mu$ then (1) has at least one positive solution.

Proof: It follows from $0 \leq f_{\infty}<\mu$ and Lemma 3.4 there exists $r>0$ such that $i\left(A, K_{r}, K\right)=1$.

By $\mu<f_{0}<\infty$ and Lemma 3.3, we can get there exists $0<\rho<r$ such that either there exists $u \in \partial K$ with $u=A u$ or $i\left(A, K_{\rho}, K\right)=0$. In the second case, $A$ has a fixed point $u \in K$ with $\rho \leq\|u\|<r$ by the properties of index. The proof is completed. 


\section{Example}

Let's consider the following boundary value problem

$$
\left\{\begin{array}{c}
D_{0+}^{\frac{3}{2}} u(t)+f(t, u(t))=0 \quad 0<t<1 \\
u^{\prime}(0)-\frac{1}{5} u\left(\frac{1}{4}\right)=0, u^{\prime}(1)+\frac{1}{5} u\left(\frac{3}{4}\right)=0
\end{array}\right.
$$

where $f(t, u)=\left\{\begin{array}{ll}\sqrt{u}+\sqrt{t}, & t \in[0,1] \text { is a irrational number } \\ 0, & t \in[0,1] \text { is a rational number }\end{array}\right.$.

Corresponding to the problem (1), we have that $\alpha=\frac{3}{2}, \xi=\frac{1}{4}, \eta=\frac{3}{4}, \beta=\frac{1}{5}, \gamma=\frac{1}{5}$. Let

$E=\{t \mid t \in[0,1]$ is a rational number $\}$, then $m(E)=0$. Obviously, (H1)-(H3) are satisfied. By simple calculation, we get $f_{0}=\infty, f_{\infty}=0$. By Theorem 3.1, we get that (6) has at least one solution. This problem can be not solved by the theorem in [11].

\section{Acknowledgements}

This work is supported by the Natural Science Foundation of China (11171088), the Natural Youth science Foundation of China (11101118) and the Natural Science Foundation of Hebei Province (A2012205074).

\section{References}

[1] Kilbas, A.A., Srivastava Hari, M. and Trujillo Juan, J. (2006) Theory and Applications of Fractional Differential Equations. North-Holland Mathematics Studied, Vol. 204. Elsevier Science BV, Amsterdam.

[2] Oldham, K.B. and Spanier, J. (1974) The Fractional Calculus. Academic Press, New York, London.

[3] Ross, B. (1975) The Fractional Calculus and Its Applications. Lecture Notes in Mathematics, Vol. 475, Springer, Berlin.

[4] Nonnenmacher, T.F. and Metzler, R. (1995) On the Riemann-Liouvile Fractional Calculus and Some Recent Applications. Fractals, 3, 557-566. http://dx.doi.org/10.1142/S0218348X95000497

[5] Tatom, F.B. (1995) The Relationship between Fractional Calculus and Fractals. Fractals, 3, 217-229. http://dx.doi.org/10.1142/S0218348X95000175

[6] Podlubny, I. (1999) Fractional Differential Equations. Mathematics in Science and Engineering, Vol. 198, AcademicPress, NewYork/London/Toronto.

[7] Samko, S.G., Kilbas, A.A. and Marichev, O.I. (1993) Fractional Integrals and Derivatives. (Theory and Applications). Gordon and Breach, Switzerland.

[8] Baleanu, D., Mustafa, O.G. and Agarwal, R.P. (2010) An Existence Result for a Super Linear Fractional Differential Equation. Applied Mathematics Letters, 23, 1129-1132. http://dx.doi.org/10.1016/j.aml.2010.04.049

[9] Baleanu, D., Agarwal, R.P., Mustafa, O.G. and Cosulschi, M. (2011) Asymptotic Integration of Some Nonlinear Differential Equations with Fractional Time Derivative. Journal of Physics A: Mathematical and Theoretical, 44. http://dx.doi.org/10.1088/1751-8113/44/5/055203

[10] Baleanu, D., Mustafa, O.G. and Agarwal, R.P. (2010) On the Solution Set for a Class of Sequential Fractional Differential Equations. Journal of Physics A: Mathematical and Theoretical, 43.

[11] Zhao, X.K., Chai, C.W. and Ge, W.G. (2011) Positive Solutions for Fractional Four-Point Boundary Value Problems. Communications in Nonlinear Science and Numerical Simulation, 16, 3665-3672. http://dx.doi.org/10.1016/j.cnsns.2011.01.002

[12] Wang, J.H., Xiang, H.J. and Liu, Z.G. (2010) Positive Solution to Nonzero Boundary Value Problem for a Coupled System of Nonlinear Fractional Differential Equations. International Journal of Differential Equations.

[13] Ahmad, B. and Nieto, J.J. (2009) Existence Results for a Coupled System of Nonlinear Fractional Differential Equations with Three-Point Boundary Conditions. Computers \& Mathematics with Applications, 58, 1838-1843. http://dx.doi.org/10.1016/j.camwa.2009.07.091

[14] Zhang, S.Q. (2006) Positive Solutions for Boundary-Value Problem of Fractional Order. Acta Mathematica Scientia, 36, 1-12. 
[15] Guo, D. and Lakshmikantham, V. (1988) Nonlinear Problem in Abstract Cones. Academic Press, San Diego.

[16] Webb, J.R.L. and Lan, K.Q. (2006) Eigenvalue Criteria for Existence of Multiple Positive Solutions of Nonlinear Boundary Value Problems of Local and Nonlocal Type. Topological Methods in Nonlinear Analysis, Journal of the Juliusz Schauder Center, 27, 91-115. 